\title{
AMENDMENTS
}

\section{Author Correction: Alveolar macrophages are epigenetically altered after inflammation, leading to long-term lung immunoparalysis}

Antoine Roquilly (D), Cedric Jacqueline (D), Marion Davieau, Alice Mollé, Abderrahmane Sadek, Cynthia Fourgeux, Paul Rooze, Alexis Broquet, Barbara Misme-Aucouturier, Tanguy Chaumette (D), Mickael Vourc'h, Raphael Cinotti, Nadege Marec, Vanessa Gauttier, Hamish E. G. McWilliam (D), Frederic Altare, Jeremie Poschmann (D), Jose A. Villadangos (D) and Karim Asehnoune (iD)

Correction to: Nature Immunology https://doi.org/10.1038/s41590-020-0673-x, published online 18 May 2020.

In the version of this article initially published, in the Methods section "In vitro phagocytosis assay", the clone number for the anti-SIPRP $\alpha$ blocking antibody was incorrectly given as OSE-172. The correct nomenclature for this antibody is "humanized blocking anti-SIRP $\alpha$ clone 18D5". The error has been corrected in the HTML and PDF versions of the article.

Published online: 24 June 2020

https://doi.org/10.1038/s41590-020-0739-9

(c) The Author(s), under exclusive licence to Springer Nature America, Inc. 2020

\section{Author Correction: $\mathrm{Cxcl} 10^{+}$monocytes define a pathogenic subset in the central nervous system during autoimmune neuroinflammation}

Amir Giladi (D), Lisa Katharina Wagner, Hanjie Li, Dorothea Dörr, Chiara Medaglia, Franziska Paul, Anat Shemer, Steffen Jung (D), Simon Yona, Matthias Mack, Achim Leutz D, Ido Amit (D) and Alexander Mildner (D)

Correction to: Nature Immunology https://doi.org/10.1038/s41590-020-0661-1, published online 20 April 2020.

In the supplementary information originally posted for this article, Supplementary Table 2 was missing the first row, which contains a color key for the corresponding gene clusters in Extended Data Fig 2. The error has been corrected online.

Published online: 8 June 2020

https://doi.org/10.1038/s41590-020-0722-5

๑ The Author(s), under exclusive licence to Springer Nature America, Inc. 2020

\section{Publisher Correction: $\mathrm{Cxc} / 10^{+}$monocytes define a pathogenic subset in the central nervous system during autoimmune neuroinflammation}

Amir Giladi D, Lisa Katharina Wagner, Hanjie Li, Dorothea Dörr, Chiara Medaglia, Franziska Paul, Anat Shemer, Steffen Jung (D), Simon Yona, Matthias Mack, Achim Leutz (D), Ido Amit (D) and Alexander Mildner (iD)

Correction to: Nature Immunology https://doi.org/10.1038/s41590-020-0661-1, published online 20 April 2020.

In the supplementary information originally posted for this article, in Supplementary Table 2, the data for the top 60 differentially expressed genes of IFN type I monocytes were missing. The error has been corrected online. In addition, the table now contains a color code for the monocyte subsets that matches the color code used throughout the article.

Published online: 24 June 2020

https://doi.org/10.1038/s41590-020-0742-1

(c) The Author(s), under exclusive licence to Springer Nature America, Inc. 2020 